\title{
'Minimal symptom expression' in patients with acetylcholine receptor antibody-positive refractory generalized myasthenia gravis treated with eculizumab
}

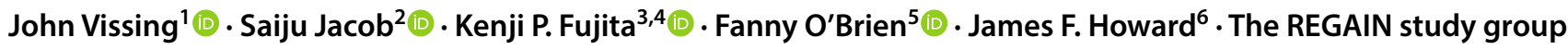

Received: 12 November 2019 / Revised: 18 February 2020 / Accepted: 20 February 2020 / Published online: 18 March 2020

(c) The Author(s) 2020

\begin{abstract}
Background The efficacy and tolerability of eculizumab were assessed in REGAIN, a 26-week, phase 3, randomized, doubleblind, placebo-controlled study in anti-acetylcholine receptor antibody-positive $(\mathrm{AChR}+)$ refractory generalized myasthenia gravis (gMG), and its open-label extension.

Methods Attainment of 'minimal symptom expression' was evaluated using patient-reported outcome measures of gMG symptoms [MG activities of daily living scale (MG-ADL), 15-item MG quality of life questionnaire (MG-QOL15)] at the completion of REGAIN and during the open-label extension. 'Minimal symptom expression' was defined as MG-ADL total score of $0-1$ or MG-QOL15 total score of 0-3.

Results At REGAIN week 26, more eculizumab-treated patients achieved 'minimal symptom expression' versus placebo [MG-ADL: $21.4 \%$ vs 1.7\%; difference 19.8\%; 95\% confidence interval (CI) 8.5, 31.0; $p=0.0007$; MG-QOL15: 16.1\% vs 1.7\%; difference 14.4\%; 95\% CI 4.3, 24.6; $p=0.0069]$. During the open-label extension, the proportion of patients in the placebo/eculizumab group who achieved 'minimal symptom expression' increased after initiating eculizumab treatment and was sustained through 130 weeks of open-label eculizumab (MG-ADL: 1.7 to 27.8\%; MG-QOL15: 1.7 to 19.4\%). At extension study week 130, similar proportions of patients in the eculizumab/eculizumab and placebo/eculizumab groups achieved 'minimal symptom expression' (MG-ADL: 22.9\% and 27.8\%, respectively, $p=0.7861$; MG-QOL15: 14.3\% and $19.4 \%$, respectively, $p=0.7531$ ). The long-term tolerability of eculizumab was consistent with previous reports.

Conclusions Patients with AChR+ refractory gMG who receive eculizumab can achieve sustained 'minimal symptom expression' based on patient-reported outcomes. 'Minimal symptom expression' may be a useful tool in measuring therapy effectiveness in gMG.
\end{abstract}

Trial registration ClinicalTrials.gov NCT01997229, NCT02301624.

Keywords Eculizumab $\cdot$ Refractory $\cdot$ Myasthenia gravis $\cdot$ Minimal symptom expression $\cdot$ Acetylcholine receptor

All members of the REGAIN study group are listed in the

"Acknowledgements" section.

Electronic supplementary material The online version of this article (https://doi.org/10.1007/s00415-020-09770-y) contains supplementary material, which is available to authorized users.

John Vissing

john.vissing@ regionh.dk

1 Department of Neurology, Copenhagen Neuromuscular Center, Rigshospitalet, University of Copenhagen, Blegdamsvej 9, 2100 Copenhagen, Denmark

2 Queen Elizabeth Neuroscience Centre and Wellcome Trust Clinical Research Facility, University Hospitals Birmingham NHS Foundation Trust, Mindelsohn Way, Edgbaston, Birmingham B15 2WB, UK
3 Alnylam Pharmaceuticals, 675 West Kendall Street, Cambridge, MA 02142, USA

4 Formerly Alexion Pharmaceuticals, 121 Seaport Boulevard, Boston, MA 02210, USA

5 Alexion Pharmaceuticals, 121 Seaport Boulevard, Boston, MA 02210, USA

6 Department of Neurology, University of North Carolina, 170 Manning Drive, Chapel Hill, NC 27599-7025, USA 


\section{Introduction}

Generalized myasthenia gravis (gMG) is an autoimmune disorder characterized by muscle weakness that worsens with muscle use [1, 2]. Symptoms associated with gMG include muscle weakness resulting in dysarthria, dysphagia, dyspnoea and fatigue in the muscles of the face, neck, arms, hands and legs [3]. Although there is no generally recognized standard definition of 'refractory' disease in gMG, criteria for refractory disease that have been used include failure to respond to conventional treatments such as immunosuppressive therapies (ISTs), inability to reduce IST use without clinical relapse, intolerable adverse reactions to conventional treatments, requirement for large doses of potentially harmful agents such as ISTs, presence of comorbidities that contraindicate conventional treatments, requirement for repeated short-term rescue therapy (e.g. intravenous immunoglobulin and plasma exchange) and recurrent myasthenic crises [1, 4-7]. As a consequence of their continued disease symptoms and persistent morbidities, patients with refractory gMG experience a heavy clinical burden [4], which severely impairs their quality of life (QOL) [8].

More than $70 \%$ of patients with gMG produce autoantibodies directed against acetylcholine receptor (AChR); these patients are classed as being $\mathrm{AChR}+$. The presence of these antibodies leads to reduced binding of the neurotransmitter acetylcholine to its receptor, accelerated degradation of AChRs and activation of the complement cascade [9-11]. Complement activation results in the cleavage of the terminal complement protein $\mathrm{C} 5$ into $\mathrm{C} 5 \mathrm{a}$ and $\mathrm{C} 5 \mathrm{~b}$ by the $\mathrm{C} 5$ convertase enzyme complexes, thus activating the terminal complement cascade [12]. The combination of accelerated AChR degradation and the complement cascade results in structural damage to the neuromuscular junction, contributing to impaired neurotransmission and the muscle weakness characteristic of gMG [9].

The humanized monoclonal antibody eculizumab specifically binds to and inhibits cleavage of C5 [12]. The phase 3, randomized, placebo-controlled REGAIN study demonstrated the efficacy and tolerability of eculizumab in AChR+refractory gMG during 6 months of therapy (NCT01997229) [13]. An interim analysis of the open-label extension of REGAIN found that eculizumab remained effective and well tolerated for up to 3 years of extended treatment (NCT02301624) [14]. During these studies, key efficacy endpoint assessments included the patient-reported MG activities of daily living scale (MGADL) [15] and the 15-item MG quality of life questionnaire (MG-QOL15) [16].

Current definitions of minimal symptoms in MG rely on physician evaluation. There are currently no definitions of minimal symptoms based exclusively on patients' assessments of their symptoms and QOL; this type of measurement could potentially be more meaningful for patients than physician-based evaluations. In a validation study for the MG-QOL15, patients in remission had a mean MGQOL15 total score of 3.3 (standard deviation, 4.4), with a range of 0-15 [17]. Remission was defined as an MG composite score of 0 and a score of 0 on either the MG-ADL or the MG manual muscle test, with the exception that an eye closure score of 1 (mild weakness) was permitted [17].

For this analysis, we adapted this previous definition of remission [17] to develop the concept of 'minimal symptom expression', using the patient-reported measures of MGADL and MG-QOL15 that were used in REGAIN and the open-label extension study. This is the first analysis of its kind to use 'minimal symptom expression' as an efficacy endpoint in gMG.

\section{Methods}

\section{Study design and participants}

The efficacy and tolerability of eculizumab were assessed in a 6-month (26-week), phase 3, randomized, placebocontrolled study of patients with $\mathrm{AChR}+$ refractory $\mathrm{gMG}$ aged 18 years or older (REGAIN) [13]. The first patient was enrolled on 30 April 2014. Eligible patients had confirmed $\mathrm{AChR}+\mathrm{gMG}$; had an MG-ADL total score of at least 6; and had received at least two ISTs, or at least one IST with intravenous immunoglobulin or plasma exchange treatment at least four times in 12 months without symptom control. Exclusion criteria included ocular-only MG symptoms [Myasthenia Gravis Foundation of America (MGFA) class I] or myasthenic crisis at screening (MGFA class V). Full eligibility criteria have been published previously [13]. Patients could enrol in the open-label extension study in the 2 weeks after completing REGAIN to receive open-label eculizumab for up to a maximum of 4 years. The extension study was completed in January 2019 [14].

At least 2 weeks before starting study treatment, patients were vaccinated against Neisseria meningitidis. Patients who were not vaccinated at the appropriate time received prophylactic antibiotics until 2 weeks after vaccination. During the open-label extension study, when appropriate according to local guidelines, patients were revaccinated against N. meningitidis. During REGAIN, patients who previously received ISTs were required to maintain their pre-study dose and schedule. During the open-label extension of REGAIN, modifications to IST dose and schedule were permitted at the study investigator's discretion.

All patients provided written, informed consent. Independent ethics committees or institutional review boards 
provided written approval for the study protocols and all amendments. The studies are registered at www.clinicaltr ials.gov.

\section{Study treatment dosing and scheduling}

During REGAIN, patients randomized to eculizumab received an induction dose of $900 \mathrm{mg}$ of eculizumab on day 1 and at weeks 1, 2 and 3, followed by a maintenance dose of $1200 \mathrm{mg}$ of eculizumab at week 4 and every 2 weeks thereafter [13]. Placebo was administered using the same schedule. All patients who continued into the open-label extension study from REGAIN underwent a 4-week blinded induction phase. During this phase, patients who had received eculizumab during REGAIN received eculizumab $1200 \mathrm{mg}$ on day 1 and at week 2, and placebo at weeks 1 and 3 (eculizumab/eculizumab group). Patients who had received placebo during REGAIN received eculizumab $900 \mathrm{mg}$ on day 1 and at weeks 1, 2 and 3 (placebo/eculizumab group). All patients received open-label eculizumab $1200 \mathrm{mg}$ at week 4 and every 2 weeks thereafter.

\section{Assessments}

The objective of REGAIN and the open-label extension study was to assess the tolerability of eculizumab and its efficacy, as measured by change in MG-ADL total score from each study's baseline. This sub-analysis evaluated the achievement of 'minimal symptom expression' in both studies, defined as achievement of an MG-ADL total score of 0-1 (range 0-24) or an MG-QOL15 total score of 0-3 (range 0-60).

The proportions of patients achieving 'minimal symptom expression' were calculated for the eculizumab and placebo treatment groups at week 26 of REGAIN and up to week
130 of the open-label extension (a total of 156 weeks of eculizumab treatment for the eculizumab/eculizumab group and 130 weeks of eculizumab treatment for the placebo/ eculizumab group). Achievement of a clinically meaningful quantitative $\mathrm{MG}$ (QMG) response, defined as an improvement of at least 5 points in QMG total score, during the study was also recorded.

Adverse events were reported and coded by preferred term using the Medical Dictionary for Regulatory Activities version 20.1. MG exacerbations, rescue therapy use and discontinuations because of adverse events were also recorded.

\section{Statistical analysis}

The significance of differences between groups was evaluated by calculating $p$ values based on Fisher's exact test for categorical variables and a two-sample $t$-test for continuous variables.

\section{Results}

\section{Patient demographics and characteristics}

Data are reported from the REGAIN study and its openlabel extension for up to a maximum total of 156 weeks of eculizumab treatment. Of the 118 patients who completed REGAIN, 117 patients continued into the open-label study (eculizumab/eculizumab $n=56$, placebo/eculizumab $n=61$; Fig. 1) and were included in the efficacy and safety analyses. Patient demographics and characteristics were similar for the eculizumab/eculizumab and placebo/eculizumab groups, with the exception that there was a greater proportion of Asian patients in the placebo/eculizumab group (Table 1).
Fig. 1 Patient disposition in REGAIN and the open-label study

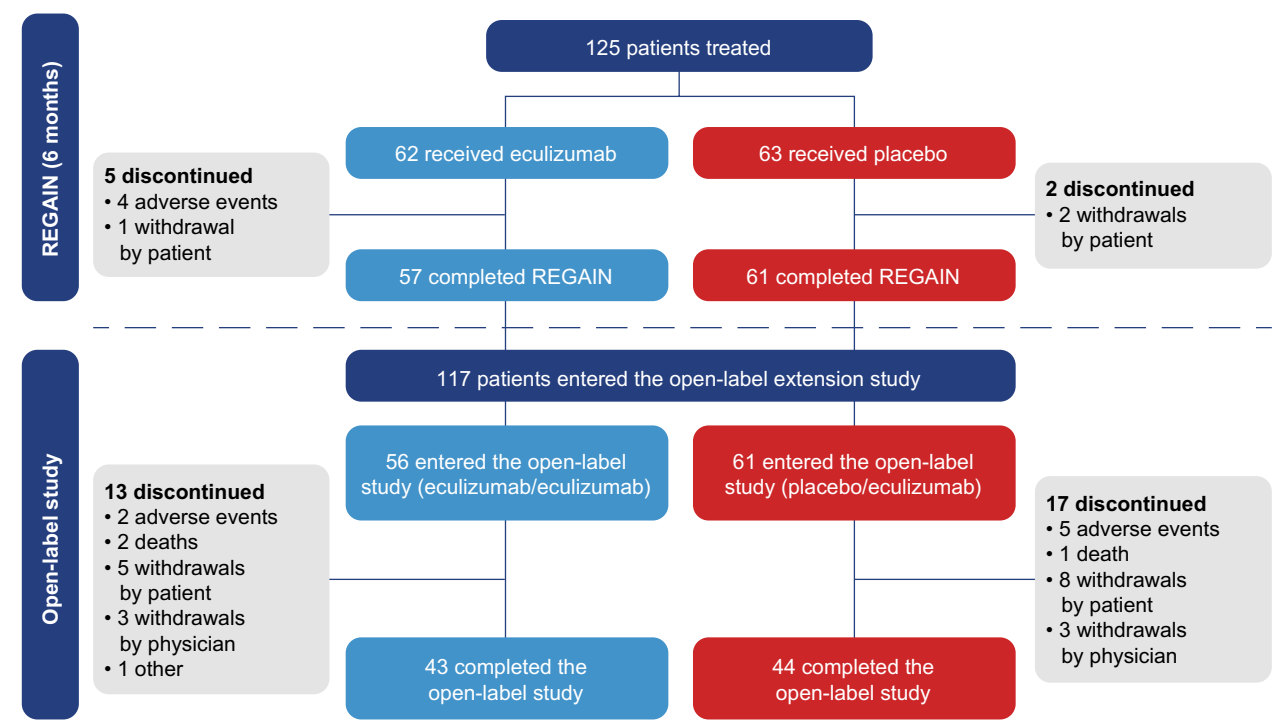


Table 1 Demographics and characteristics at REGAIN baseline of patients who continued from REGAIN into the open-label extension study

\begin{tabular}{|c|c|c|c|}
\hline Variable & $\begin{array}{l}\text { Eculizumab/ecu- } \\
\text { lizumab } n=56\end{array}$ & $\begin{array}{l}\text { Placebo/eculi- } \\
\text { zumab } n=61\end{array}$ & All patients $N=117$ \\
\hline Age, years ${ }^{\mathrm{a}}$, mean $(\mathrm{SD})$ & $46.8(15.6)$ & $47.0(17.8)$ & $46.9(16.7)$ \\
\hline \multicolumn{4}{|l|}{ Sex, $n(\%)$} \\
\hline Male & $18(32.1)$ & $20(32.8)$ & $38(32.5)$ \\
\hline Female & $38(67.9)$ & $41(67.2)$ & $79(67.5)$ \\
\hline \multicolumn{4}{|l|}{ Race, $n(\%)$} \\
\hline Asian & $3(5.4)$ & $16(26.2)$ & $19(16.2)$ \\
\hline Black or African-American & $0(0.0)$ & $2(3.3)$ & $2(1.7)$ \\
\hline White & $47(83.9)$ & $41(67.2)$ & $88(75.2)$ \\
\hline Other/multiple/unknown & $6(10.7)$ & $2(3.3)$ & $8(6.8)$ \\
\hline Duration of $\mathrm{MG}^{\mathrm{b}}$, years, mean (SD) & $10.2(7.9)$ & $9.2(8.6)$ & $9.7(8.2)$ \\
\hline Baseline MG-ADL total score, mean (SD) & $10.3(3.0)$ & $9.9(2.6)$ & $10.1(2.8)$ \\
\hline Baseline MG-QOL15 total score, mean (SD) & $32.5(12.0)$ & $30.8(12.9)$ & $31.6(12.5)$ \\
\hline
\end{tabular}

$M G$ myasthenia gravis, $M G-A D L$ myasthenia gravis activities of daily living questionnaire, $M G-Q O L 15$ 15 -item myasthenia gravis quality of life questionnaire, $S D$ standard deviation

${ }^{a}$ At first dose in REGAIN

${ }^{\mathrm{b}}$ Time from MG diagnosis to date of first dose in REGAIN

\section{'Minimal symptom expression' status during REGAIN}

At week 26 of REGAIN, a significantly higher proportion of patients receiving eculizumab achieved 'minimal symptom expression' than of those receiving placebo according to MG-ADL score $(21.4 \%$ and $1.7 \%$, respectively; difference 19.8\%; 95\% confidence interval [CI] 8.5, 31.0; $p=0.0007$; Fig. 2a) and MG-QOL15 score (16.1\% and $1.7 \%$, respectively; difference $14.4 \%$; 95\% CI 4.3, 24.6; $p=0.0069$; Fig. 2b).

\section{'Minimal symptom expression' status during the open-label study}

During the open-label extension, the proportion of patients in the eculizumab/eculizumab group with 'minimal symptom expression' was maintained for 2.5 years, between REGAIN week 26 and open-label week 130 (MG-ADL: $21.4 \%$ and $22.9 \%$, respectively; MG-QOL15: $16.1 \%$ and $14.3 \%$, respectively). In the placebo/eculizumab group, the proportion of patients with 'minimal symptom expression' increased to levels similar to those in the eculizumab/ eculizumab group in the 4 weeks after starting open-label eculizumab therapy, between REGAIN week 26 and openlabel week 4 (MG-ADL: $1.7 \%$ and $21.3 \%$, respectively; MG-QOL15: $1.7 \%$ and $17.2 \%$, respectively). This increase was sustained to open-label week 130 (MG-ADL: 27.8\%; MG-QOL15: 19.4\%).

At week 130 of the open-label extension, 'minimal symptom expression' was achieved by similar proportions of patients in the eculizumab/eculizumab and placebo/eculizumab groups as assessed by MG-ADL score $(22.9 \%$ and
$27.8 \%$, respectively; difference $-4.9 \%$; $95 \%$ CI $-25.1,15.3$; $p=0.7861$; Fig. 2a). The proportions of patients achieving 'minimal symptom expression' at week 130 based on MGQOL15 score were also similar in the two groups, being $14.3 \%$ in the eculizumab/eculizumab group and $19.4 \%$ in the placebo/eculizumab group (difference $-5.2 \%$; $95 \%$ CI $-22.5,12.2 ; p=0.7531$; Fig. 2b). Overall, $25.4 \%$ of eculizumab-treated patients experienced 'minimal symptom expression' according to MG-ADL and $16.9 \%$ according to MG-QOL15 at this time point.

Most eculizumab-treated patients who achieved 'minimal symptom expression' at any time also experienced a clinically meaningful improvement in physician-reported QMG total score, defined as an improvement of at least 5 points from eculizumab start. For 'minimal symptom expression' according to MG-ADL total score, this proportion was $85.7 \%$ (42/49) and, for 'minimal symptom expression' according to MG-QOL15 total score, it was $81.1 \%$ (30/37).

There was no significant difference in mean age at first eculizumab dose between eculizumab-treated patients who achieved 'minimal symptom expression' according to MG-ADL at any time during REGAIN and the open-label study (up to week 130) and those who did not (47.4 vs 47.0 years; $p=0.8847$ ). Mean disease duration at first eculizumab dose was shorter for patients who achieved 'minimal symptom expression' according to MG-ADL by open-label week 130 than for those who did not $[8.27$ (range 1.6-27.0) vs 11.16 (range 1.7-34.4) years; $p=0.0474]$. For achievement of 'minimal symptom expression' according to MG-QOL15 up to openlabel week 130, there were no significant differences in mean age ( 44.6 vs 48.4 years; $p=0.2611$ ) or mean disease 

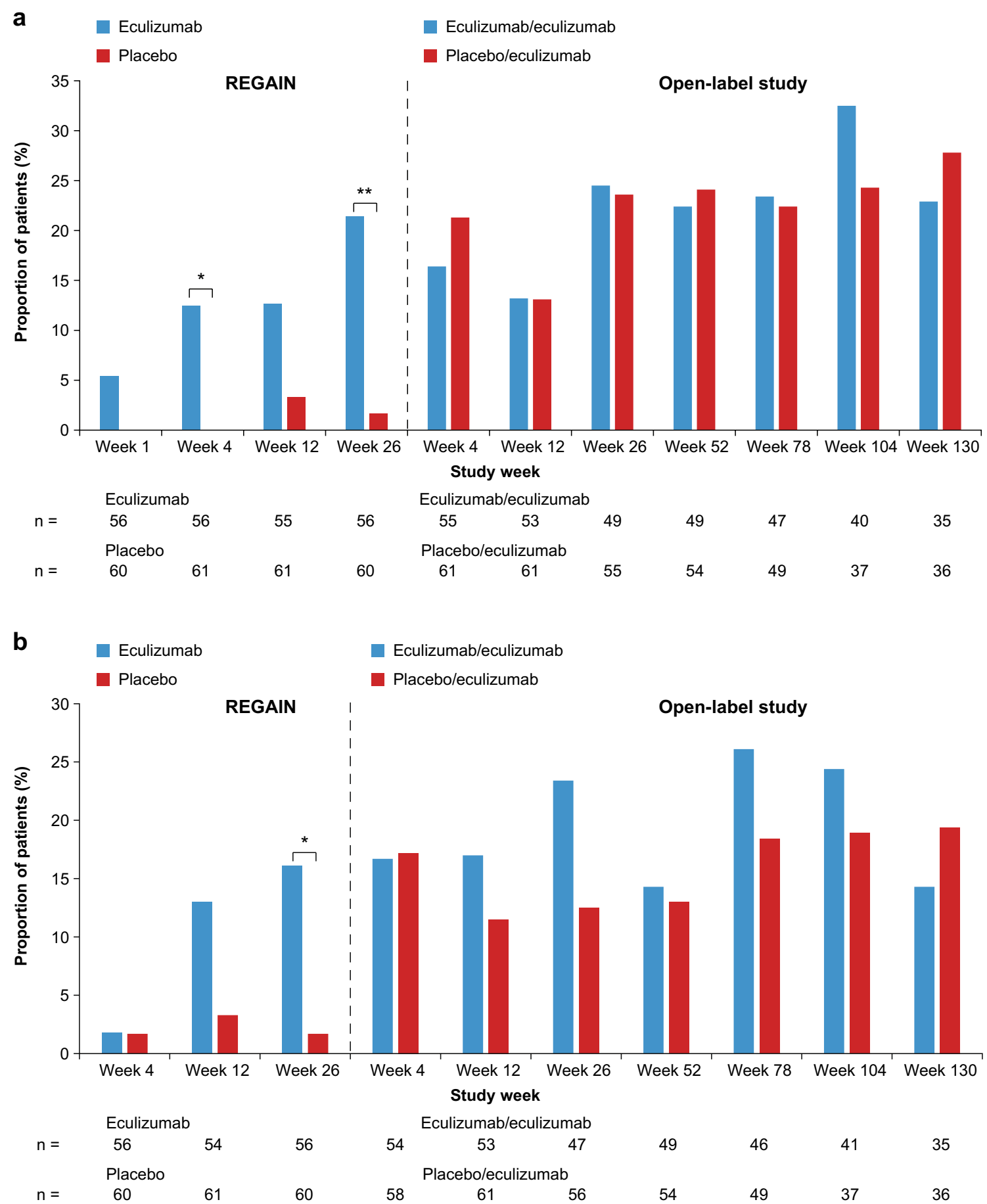

Fig. 2 a Proportions of patients achieving 'minimal symptom expression', defined as an MG-ADL total score of 0-1. b Proportions of patients achieving 'minimal symptom expression', defined as an MGQOL15 total score of $0-3 .{ }^{*} p<0.01 ;{ }^{* *} p<0.001$ vs placebo. $p$ values are based on Fisher's exact test. $M G-A D L$ myasthenia gravis activities of daily living questionnaire, $M G$ - $Q O L 15$ 15-item myasthenia gravis quality of life questionnaire

either MG-ADL or MG-QOL15 scores, in other baseline characteristics, including sex, race, MGFA class, history of MG crisis and history of IST use. The only significant differences in baseline MG-ADL, MG-QOL15 and QMG not achieve 'minimal symptom expression', according to 
total scores were for MG-ADL $(p=0.0380)$ and MGQOL15 $(p=0.0487)$ between patients who did achieve 'minimal symptom expression' according to MG-QOL15 and those who did not (Table 2).

The mean MG-ADL total score for the open-label study population decreased from 10.1 [standard deviation (SD) 2.80; $n=117$ ] at REGAIN baseline to 3.9 (SD 3.08; $n=71$ ) at open-label week 130. The mean MG-QOL15 total score also reduced between these time points, from 31.6 (SD 12.48) to 15.3 (SD 12.15).

\section{Safety}

Safety data have previously been published for REGAIN and an interim analysis of the open-label extension study [13, 14]. During these two studies, headache and nasopharyngitis were the most common adverse events among patients receiving eculizumab (experienced by $44.4 \%$ and $38.5 \%$, respectively, from REGAIN baseline to week 130 of the open-label extension). MG worsening was experienced by $15.4 \%$ of eculizumab-treated patients, MG crisis by $3.4 \%$

Table 2 Baseline demographics and characteristics of patients who did or did not achieve 'minimal symptom expression' at any time during REGAIN and the open-label extension study

\begin{tabular}{|c|c|c|c|c|c|c|}
\hline \multirow[t]{2}{*}{ Variable } & \multicolumn{3}{|c|}{ MG-ADL total score $0-1$} & \multicolumn{3}{|c|}{ MG-QOL15 total score $0-3$} \\
\hline & Did achieve $n=49$ & Did not achieve $n=68$ & $p$ value $^{\mathrm{a}}$ & Did achieve $n=37$ & Did not achieve $n=80$ & $p$ value $^{\mathrm{a}}$ \\
\hline \multicolumn{7}{|l|}{ Sex, $n(\%)$} \\
\hline Male & $14(28.6)$ & $24(35.3)$ & \multirow[t]{2}{*}{0.5491} & $11(29.7)$ & $27(33.8)$ & \multirow[t]{2}{*}{0.8322} \\
\hline Female & $35(71.4)$ & $44(64.7)$ & & $26(70.3)$ & $53(66.3)$ & \\
\hline \multicolumn{7}{|l|}{ Race, $n(\%)$} \\
\hline Asian & $7(14.3)$ & $12(17.6)$ & \multirow[t]{4}{*}{0.5767} & $5(13.5)$ & $14(17.5)$ & \multirow[t]{4}{*}{0.3377} \\
\hline Black or African American & $1(2.0)$ & $1(1.5)$ & & $1(2.7)$ & $1(1.3)$ & \\
\hline White & $39(79.6)$ & $49(72.1)$ & & $28(75.7)$ & $60(75.0)$ & \\
\hline Other/multiple/unknown & $2(4.1)$ & $6(8.8)$ & & $3(8.1)$ & $5(6.3)$ & \\
\hline $\begin{array}{l}\text { Age at first eculizumab dose, years, } \\
\text { mean (SD) }\end{array}$ & $47.4(18.79)$ & $47.0(15.25)$ & 0.8847 & $44.6(19.23)$ & $48.4(15.45)$ & 0.2611 \\
\hline $\begin{array}{l}\text { Duration of MG at first eculizumab } \\
\text { dose }^{b} \text {, years, mean (SD) }\end{array}$ & $8.3(6.57)$ & $11.2(9.08)$ & 0.0474 & $8.7(5.97)$ & $10.5(9.05)$ & 0.2091 \\
\hline $\begin{array}{l}\text { MG-ADL total score at REGAIN } \\
\text { baseline, mean (SD) }\end{array}$ & $9.6(3.08)$ & $10.4(2.55)$ & 0.1061 & $9.3(2.79)$ & $10.5(2.75)$ & 0.0380 \\
\hline $\begin{array}{l}\text { MG-QOL15 total score at REGAIN } \\
\text { baseline, mean (SD) }\end{array}$ & $31.0(13.23)$ & $32.0(12.00)$ & 0.6709 & $28.2(14.14)$ & $33.1(11.40)$ & 0.0487 \\
\hline $\begin{array}{l}\text { QMG total score at REGAIN base- } \\
\text { line, mean (SD) }\end{array}$ & $16.8(5.51)$ & $17.1(5.21)$ & 0.8247 & $17.1(5.77)$ & $16.9(5.13)$ & 0.9034 \\
\hline \multicolumn{7}{|c|}{ Patients with MGFA class at REGAIN screening, $n(\%)$} \\
\hline IIa & $10(20.4)$ & $14(20.6)$ & \multirow[t]{6}{*}{0.7087} & $10(27.0)$ & $14(17.5)$ & \multirow[t]{6}{*}{0.7954} \\
\hline IIb & $11(22.4)$ & $8(11.8)$ & & $7(18.9)$ & $12(15.0)$ & \\
\hline IIIa & $13(26.5)$ & $21(30.9)$ & & $10(27.0)$ & $24(30.0)$ & \\
\hline IIIb & $10(20.4)$ & $18(26.5)$ & & $8(21.6)$ & $20(25.0)$ & \\
\hline IVa & $2(4.1)$ & $4(5.9)$ & & $1(2.7)$ & $5(6.3)$ & \\
\hline $\mathrm{IVb}$ & $3(6.1)$ & $3(4.4)$ & & $1(2.7)$ & $5(6.3)$ & \\
\hline $\begin{array}{l}\text { Patients with history of MG crisis } \\
\text { before REGAIN, } n(\%)\end{array}$ & $8(16.3)$ & $13(19.1)$ & 0.8091 & $6(16.2)$ & $15(18.8)$ & 0.8018 \\
\hline \multicolumn{7}{|c|}{ Patients using ISTs before REGAIN, $n(\%)$} \\
\hline 1 IST & $0(0.0)$ & $2(2.9)$ & \multirow[t]{4}{*}{0.1818} & $0(0.0)$ & $2(2.5)$ & \multirow[t]{4}{*}{0.0520} \\
\hline 2 ISTs & $27(55.1)$ & $26(38.2)$ & & $22(59.5)$ & $31(38.8)$ & \\
\hline 3 ISTs & $15(30.6)$ & $23(33.8)$ & & $12(32.4)$ & $26(32.5)$ & \\
\hline$\geq 4$ ISTs & $7(14.3)$ & $17(25.0)$ & & $3(8.1)$ & $21(26.3)$ & \\
\hline
\end{tabular}

$I S T$ immunosuppressive therapy, $M G$ myasthenia gravis, $M G$ - $A D L$ myasthenia gravis activities of daily living scale, $M G F A$ Myasthenia Gravis Foundation of America, $M G-Q O L 15$ 15-item myasthenia gravis quality of life questionnaire, $Q M G$ quantitative myasthenia gravis scale, $S D$ standard deviation

${ }^{a}$ The significance of differences between groups was evaluated by calculating $p$ values based on Fisher's exact test for categorical variables and a two-sample $t$-test for continuous variables

${ }^{\mathrm{b}}$ Time from MG diagnosis to date of first eculizumab dose 
and MG exacerbations by $29.1 \%$. A total of 11 patients discontinued eculizumab therapy owing to adverse events during the two studies. One patient contracted a meningococcal infection, which was resolved with antibiotic treatment [13]. Three deaths were reported in patients with important comorbidities that were likely to have contributed to the clinical outcome [13].

\section{Discussion}

This analysis found that, at the end of REGAIN, a significantly greater proportion of patients with $\mathrm{AChR}+$ refractory gMG treated with eculizumab experienced 'minimal symptom expression' than of those receiving placebo according to an MG-ADL total score of 0-1 or an MG-QOL15 total score of $0-3$. The proportions of patients experiencing 'minimal symptom expression' were maintained through 2.5 years of open-label eculizumab therapy in the extension study.

The only significant difference in baseline characteristics between patients who did and did not achieve 'minimal symptom expression' according to MG-ADL was in disease duration, and the only significant differences in the achievement of 'minimal symptom expression' according to MGQOL15 were in MG-ADL and MG-QOL15 total scores at REGAIN baseline. The difference in baseline MG-ADL total score between these groups was small (1.2) and not clinically relevant. The baseline MG-QOL15 score was 4.9 points lower in patients who did achieve 'minimal symptom expression' according to MG-QOL15 than in those who did not, which may simply reflect that less improvement was required for patients with a lower baseline MG-QOL15 score to achieve a score of 3 or less. Overall, patients who did achieve 'minimal symptom expression' did not have less severe disease before eculizumab treatment than those who did not achieve it.

It is notable that, among a group of patients with refractory gMG with a mean MG-ADL total score of 10.1 at the start of REGAIN, approximately a quarter reported 'minimal symptom expression' defined as an MG-ADL total score of $0-1$ through week 130 of the open-label study, by which time point the mean MG-ADL total score had reduced by more than half to 3.9. This reflects patient-reported improvements in disease burden in excess of the two-point reduction in MG-ADL total score that is considered to be a clinically meaningful improvement [18] to a level that has previously been described as disease remission [17]. In addition, 'minimal symptom expression', defined as an MG-QOL15 total score of $0-3$, was achieved by one-sixth of these patients, and the mean MG-QOL15 total score halved between the start of REGAIN (31.6) and week 130 of the open-label study (15.3). The smaller proportion achieving 'minimal symptom expression' according to MG-QOL15 versus
MG-ADL (one-sixth vs one-quarter) may be due to the conservative MG-QOL15 total score range (0-3) used in the definition of 'minimal symptom expression' in this analysis.

A correlation between changes in patient-reported MGADL scores and physician-assessed QMG scores has been described previously [19, 20]. In REGAIN and its open-label extension, patient-reported improvements were reflected in improvements in physician-reported outcomes assessed using QMG scoring. Almost half of eculizumab-treated patients achieved a clinically meaningful improvement in QMG total score (a reduction of at least 5 points) in the 26 weeks of REGAIN, and significant decreases in mean QMG total scores with eculizumab were maintained for up to 3 years during REGAIN and its open-label extension [13, 14]. In this analysis, most patients who achieved patientreported 'minimal symptom expression' also achieved a clinically meaningful physician-reported QMG response.

The long-term tolerability of eculizumab was consistent with its known adverse event profile from established indications [21-25], and no new safety signals were observed since the interim analysis of the open-label extension study [14].

The main limitation of this post hoc analysis is the openlabel design of the extension study, which could yield unconscious bias in reporting. Given that over $90 \%$ of patients who enrolled in REGAIN continued into the open-label study, selection bias in the open-label study population is unlikely. Further, the novel definition of 'minimal symptom expression' used in this analysis was derived from previous definitions of remission and has not yet been formally validated. In addition, further research is needed to evaluate the optimal range for this patient-reported assessment because this analysis used a conservative MG-QOL15 total score range of 0-3 to indicate 'minimal symptom expression'.

In conclusion, the results of this analysis confirm a rapid and sustained clinical response to eculizumab in patients with refractory gMG, reflected in the higher proportion reporting 'minimal symptom expression' with eculizumab than with placebo. Despite having refractory MG, individuals can achieve long-term 'minimal symptom expression' with eculizumab therapy. The current lack of validated definitions of minimal symptoms based exclusively on patients' assessments of their symptoms and QOL makes it difficult to comment on the generalizability of these findings. However, this type of assessment could potentially be more meaningful for patients than physician-based evaluations. 'Minimal symptom expression' based on quantitative, patient-reported outcomes may, therefore, be a useful tool in measuring patient progress following therapeutic intervention.

Acknowledgements The authors would like to thank the patients who took part in REGAIN and the open-label extension study, as well as their families. Completion of such a large, multinational study could not have been accomplished without the participation of our investigators and collaborators (a list of whom is provided in the supplementary 
materials [Online Resource 1]). We thank them for their contributions to the completion of the study. We also thank Sivani Paskaradevan and Róisín Armstrong (Alexion Pharmaceuticals) for critical review of the manuscript and Cindy Lane (formerly of Alexion Pharmaceuticals) for clinical study oversight. We would like to acknowledge Corin Wing $\mathrm{PhD}$ (Oxford PharmaGenesis, Oxford, UK), who provided medical writing support in the production of this manuscript (funded by Alexion Pharmaceuticals). All members of the REGAIN study group are listed: Argentina: Instituto de Investigaciones Neurológicas Raúl Carrea (FLENI), Buenos Aires, Argentina: Principal investigators: Claudio Gabriel Mazia; sub-investigators: Miguel Wilken, Fabio Barroso; study coordinator: Juliet Saba. Hospital Italiano de Buenos Aires, Buenos Aires, Argentina: Principal investigator: Marcelo Rugiero; sub-investigators: Mariela Bettini, Marcelo Chaves, Gonzalo Vidal; study coordinator: Alejandra Dalila Garcia. Belgium: Algemeen Ziekenhuis SintLucas - Campus Sint-Lucas, Ghent, Belgium: Principal investigator: Jan De Bleecker; sub-investigator: Guy Van den Abeele; primary study coordinator: Kathy de Koning; study coordinator: Katrien De Mey. Universitair Ziekenhuis Antwerpen, Edegem, Antwerp, Belgium: Principal investigator: Rudy Mercelis; study coordinators: Délphine Mahieu, Linda Wagemaekers. Universitaire Ziekenhuizen Leuven, Leuven, Belgium: Principal investigator: Philip Van Damme (Laboratory of Neurobiology, Department of Neuroscience, Katholieke Universiteit Leuven and Center for Brain \& Disease Research); sub-investigators: Annelies Depreitere, Caroline Schotte, Charlotte Smetcoren, Olivier Stevens, Sien Van Daele, Nicolas Vandenbussche, Annelies Vanhee, Sarah Verjans, Jan Vynckier; primary study coordinator: Ann D'Hont; study coordinator: Petra Tilkin. Brazil: Faculdade de Medicina do ABC, Santo André, Brazil: Principal investigator: Alzira Alves de Siqueira Carvalho; sub-investigators: Igor Dias Brockhausen, David Feder; study coordinators: Daniel Ambrosio, Pamela César, Ana Paula Melo, Renata Martins Ribeiro, Rosana Rocha, Bruno Bezerra Rosa, Thabata Veiga; study coordinators (back-up): Luiz Augusto da Silva, Murilo Santos Engel, Jordana Gonçalves Geraldo. Fundação Faculdade Regional de Medicina de São José do Rio Preto, São José do Rio Preto, Brazil: Principal investigator: Maria da Penha Ananias Morita; subinvestigators: Erica Nogueira Coelho, Gabriel Paiva, Marina Pozo, Natalia Prando; study coordinators: Debora Dada Martineli Torres, Cristiani Fernanda Butinhao, Gustavo Duran, Tomás Augusto Suriane Fialho, Tamires Cristina Gomes da Silva, Luiz Otavio Maia Gonçalves, Lucas Eduardo Pazetto, Luciana Renata Cubas Volpe, Luciana Souza Duca. Hospital Mãe de Deus, Porto Alegre, Brazil: Principal investigator: Maurício André Gheller Friedrich; sub-investigator: Alexandre Guerreiro, Henrique Mohr, Maurer Pereira Martins; study coordinators: Daiane da Cruz Pacheco, Luciana Ferreira, Ana Paula Macagnan, Graziela Pinto, Aline de Cassia Santos. Universidade Federal de São Paulo [UNIFESP], São Paulo, Brazil: Principal investigator: Acary Souza Bulle Oliveira; sub-investigators: Ana Carolina Amaral de Andrade, Marcelo Annes, Liene Duarte Silva, Valeria Cavalcante Lino, Wladimir Pinto; study coordinators: Natália Assis, Fernanda Carrara, Carolina Miranda, Iandra Souza; study coordinator (back-up): Patrícia Fernandes. Canada: University of Alberta Hospital, Edmonton, AB, Canada: Principal investigator: Zaeem Siddiqi; sub-investigator: Cecile Phan; primary study coordinator: Jeffrey Narayan; study coordinators; Derrick Blackmore, Ashley Mallon, Rikki Roderus, Elizabeth Watt. Czech Republic: Fakultní nemocnice Brno, Brno, Czech Republic: Principal investigator: Stanislav Vohanka; sub-investigators: Josef Bednarik, Magda Chmelikova, Marek Cierny; study coordinator: Stanislava Toncrova. Fakultní nemocnice Ostrava, Ostrava, Czech Republic: Principal investigator: Jana Junkerova; sub-investigators: Barbora Kurkova, Katarina Reguliova, Olga Zapletalova. Všeobecná fakultní nemocnice v Praze, Praze, Czech Republic: Principal investigator: Jiri Pitha; subinvestigators: Iveta Novakova, Michaela Tyblova; study coordinators: Ivana Jurajdova, Marcela Wolfova. Denmark: Århus Universitetshospital, Aarhus, Denmark: Principal investigator: Henning Andersen; sub-investigators: Thomas Harbo, Lotte Vinge; primary study coordinator: Susanne Krogh; study coordinator: Anita Mogensen. Rigshospitalet, Copenhagen, Denmark: Principal investigator: John Vissing; sub-investigators: Joan Højgaard, Nanna Witting; primary study coordinator: Anne Mette Ostergaard Autzen; study coordinator: Jane Pedersen. Finland: Neuro NEO Oy, Turku, Finland: Principal investigator: Juha-Pekka Erälinna; sub-investigator: Mikko Laaksonen; Olli Oksaranta; primary study coordinator: Tuula Harrison; study coordinator (back-up): Jaana Eriksson. Hungary: Jahn Ferenc Dél-Pesti Kórház, Budapest, Hungary: Principal investigator: Csilla Rozsa; sub-investigators: Melinda Horvath, Gabor Lovas, Judit Matolcsi, Gyorgyi Szabo; study coordinators: Gedeonne Jakab, Brigitta Szabadosne. University of Szeged, Albert Szent-Györgyi Health Center, Szeged, Hungary: Principal investigator: Laszlo Vecsei; sub-investigators: Livia Dezsi, Edina Varga; study coordinator: Monika Konyane. Italy: Azienda Ospedaliera Sant'Andrea - Università di Roma La Sapienza, Rome, Italy: Principal investigator: Giovanni Antonini; sub-investigators: Antonella Di Pasquale, Matteo Garibaldi, Stefania Morino, Fernanda Troili, Laura Fionda; primary study coordinators: Antonella Di Pasquale, Matteo Garibaldi. Azienda Ospedaliera Universitaria "Federico II", Naples, Italy: Principal investigator: Francesco Saccà; previous principal investigator: Alessandro Filla; sub-investigators; Teresa Costabile, Enrico Marano; study coordinators: Angiola Fasanaro, Angela Marsili, Giorgia Puorro. Fondazione IRCCS Istituto Neurologico Carlo Besta, Milan, Italy: Principal investigator: Renato Mantegazza; sub-investigators: Carlo Antozzi, Silvia Bonanno, Giorgia Camera, Alberta Locatelli, Lorenzo Maggi, Maria Pasanisi; study coordinator: Angela Campanella. Policlinico Universitario Agostino Gemelli, Rome, Italy: Principal investigator: Amelia Evoli; sub-investigators: Paolo Emilio Alboini, Valentina D'Amato, Raffaele Iorio. Umberto I Policlinico di Roma Università di Roma La Sapienza, Rome, Italy: Principal investigator: Maurizio Inghilleri; sub-investigators: Laura Fionda, Vittorio Frasca, Elena Giacomelli, Maria Gori, Diego Lopergolo, Emanuela Onesti; study coordinators: Vittorio Frasca, Maria Gabriele. Japan: Chiba University Hospital, Chiba, Japan: Principal investigator: Akiyuki Uzawa; sub-investigators: Tetsuya Kanai, Naoki Kawaguchi, Masahiro Mori; primary study coordinator: Yoko Kaneko; study coordinators: Akiko Kanzaki, Eri Kobayashi. Kyushu University Hospital, Fukuoka, Japan: Principal investigator: Hiroyuki Murai; sub-investigators: Katsuhisa Masaki, Dai Matsuse, Takuya Matsushita, Taira Uehara; primary study coordinator: Misa Shimpo; study coordinators: Maki Jingu, Keiko Kikutake, Yumiko Nakamura; study coordinator (back-up): Yoshiko Sano. Hanamaki General Hospital, Hanamaki, Japan: Principal investigator: Kimiaki Utsugisawa; sub-investigator: Yuriko Nagane; primary study coordinators: Ikuko Kamegamori, Tomoko Tsuda; study coordinators: Yuko Fujii, Kazumi Futono, Yukiko Ozawa, Aya Mizugami, Yuka Saito. Kinki University Hospital, Osaka, Japan: Principal investigator: Makoto Samukawa; previous principal investigator; Hidekazu Suzuki; sub-investigator: Miyuki Morikawa; primary study coordinators: Sachiko Kamakura, Eriko Miyawaki. Nagasaki University Hospital, Nagasaki, Japan: Principal investigator: Hirokazu Shiraishi; subinvestigators: Teiichiro Mitazaki, Masakatsu Motomura, Akihiro Mukaino, Shunsuke Yoshimura; primary study coordinators: Shizuka Asada, Seiko Yoshida; study coordinators: Shoko Amamoto, Tomomi Kobashikawa, Megumi Koga, Yasuko Maeda, Kazumi Takada, Mihoko Takada, Masako Tsurumaru, Yumi Yamashita, Seiko Yoshida. National Hospital Organization Sendai Medical Center, Sendai, Japan: Principal investigator: Yasushi Suzuki; sub-investigators: Tetsuya Akiyama, Koichi Narikawa, Ohito Tano, Kenichi Tsukita; primary study coordinators: Rikako Kurihara, Fumie Meguro; study coordinators: Yusuke Fukuda, Miwako Sato. Osaka University Hospital, Osaka, Japan: Principal investigator: Meinoshin Okumura; sub-investigators: Soichiro Funaka, Tomohiro Kawamura, Masayuki Makamori, Masanori Takahashi; primary study coordinator: Namie Taichi; study coordinators: Tomoya Hasuike, Eriko Higuchi, Hisako Kobayashi, Kaori Osakada. Sapporo Medical University Hospital, Chuo-ku, Sapporo, Japan: Principal investigators: Tomihiro Imai, Emiko Tsuda; previous principal 
investigator: Shun Shimohama; sub-investigators: Takashi Hayashi, Shin Hisahara, Tomihiro Imai, Jun Kawamata, Takashi Murahara, Masaki Saitoh, Shun Shimohama, Shuichiro Suzuki, Daisuke Yamamoto; primary study coordinator: Yoko Ishiyama; study coordinators: Naoko Ishiyama, Mayuko Noshiro, Rumi Takeyama, Kaori Uwasa, Ikuko Yasuda. Republic of Korea: Korea University Anam Hospital, Seoul, Republic of Korea: Principal investigator: Byung-Jo Kim; subinvestigators: Chang Nyoung Lee, Yong Seo Koo, Hung Youl Seok: study coordinators: Hoo Nam Kang, HyeJin Ra. Samsung Medical Center, Seoul, Republic of Korea: Principal investigator: Byoung Joon Kim; sub-investigators: Eun Bin Cho, MiSong Choi, HyeLim Lee, JuHong Min, Jinmyoung Seok; study coordinators: JiEun Lee, Da Yoon Koh, JuYoung Kwon, SangAe Park; study coordinator (back-up): Eun Haw Choi. Seoul National University Seoul Metropolitan Government Boramae Medical Center, Seoul, Republic ofKorea: Principal investigator: Yoon-Ho Hong; sub-investigators: So-Hyun Ahn, Dae Lim Koo, Jae-Sung Lim, Chae Won Shin; study coordinators: Ji Ye Hwang,Miri Kim. Severance Hospital, Yonsei University Health System, Seoul, Republic ofKorea: Principal investigator: Seung Min Kim; sub-investigators: Ha-Neul Jeong, JinWoo Jung, Yool-hee Kim, Hyung Seok Lee, Ha Young Shin; study coordinators: Eun Bi Hwang, Miju Shin. Netherlands: Academisch Medisch Centrum, Amsterdam, Netherlands: Principal investigator: Anneke van der Kooi; sub-investigator: Marianne de Visser; primary study coordinator: Tamar Gibson. Spain: Hospital Universitari de Bellvitge, Barcelona, Spain: Principal investigator: Carlos Casasnovas; sub-investigators: Maria Antonia Alberti Aguilo, Christian Homedes-Pedret, Natalia Julia Palacios, Laura Diez Porras, Valentina Velez Santamaria; primary study coordinator: Ana Lazaro. Hospital Universitario La Paz, Madrid, Spain: Principal investigator: Exuperio Diez Tejedor, Pilar Gomez Salcedo; sub-investigators: Mireya Fernandez-Fournier, Pedro Lopez Ruiz, Francisco Javier Rodriguez de Rivera; primary study coordinator: Mireya FernandezFournier; study coordinator: Maria Sastre. Hospital Universitari Vall d'Hebron, Barcelona, Spain: Principal investigator: Josep Gamez Carbonell, Pilar Sune; sub-investigator: Maria Salvado Figueras; primary study coordinator: Gisela Gili, Gonzalo Mazuela. Hospital Sant Pau, Universitat Autònoma de Barcelona, Spain: Principal investigator: Isabel Illa; sub-investigators: Elena Cortes Vicente, Jordi Diaz-Manera, Luis Antonio Querol Gutiérrez, Ricardo Rojas Garcia; primary study coordinator: Nuria Vidal; study coordinator (back-up): Elisabet Arribas-Ibar. Sweden: Karolinska University Hospital, Stockholm, Sweden: Principal investigator: Fredrik Piehl; sub-investigator: Albert Hietala; primary study coordinator: Lena Bjarbo. Turkey: Dokuz Eylül University Faculty of Medicine, Izmir, Turkey: Principal investigator: Ihsan Sengun; sub-investigators: Arzu Meherremova, Pinar Ozcelik; study coordinators: Bengu Balkan, Celal Tuga, Muzeyyen Ugur. Hacettepe University Faculty of Medicine, Ankara, Turkey: Principal investigator: Sevim Erdem-Ozdamar; sub-investigators: Can Ebru BekircanKurt, Nazire Pinar Acar, Ezgi Yilmaz; primary study coordinator: Yagmur Caliskan; study coordinator: Gulsah Orsel. Kocaeli University Faculty of Medicine, Kocaeli, Turkey: Principal investigator: Husnu Efendi; sub-investigators: Seda Aydinlik, Hakan Cavus, Ayse Kutlu; study coordinators: Gulsah Becerikli, Cansu Semiz, Ozlem Tun. Ondokuz Mayis University Medical Faculty, Atakum/Samsun, Turkey: Principal investigator: Murat Terzi; sub-investigators: Baki Dogan, Musa Kazim Onar, Sedat Sen; study coordinators: Tugce Kirbas Cavdar, Adife Veske. United Kingdom: King's College Hospital, London, UK: Principal investigator: Fiona Norwood; sub-investigators: Aikaterini Dimitriou, Jakit Gollogly, Mohamed Mahdi-Rogers, Arshira Seddigh, Giannis Sokratous; study coordinators: Gal Maier, Faisal Sohail. Queen Elizabeth Neuroscience Centre, (Wellcome Trust CRF, University Hospitals Birmingham), Birmingham, UK: Principal investigator: Saiju Jacob; sub-investigators: Girija Sadalage, Pravin Torane; primary study coordinators: Claire Brown, Amna Shah. The Walton Centre, Liverpool, UK: Principal investigator: Sivakumar Sathasivam; sub-investigator: Heike Arndt; primary study coordinator: Debbie Davies; study coordinator: Dave Watling. United States of America: Brigham and Women's Hospital, Boston, MA, USA: Principal investigator: Anthony Amato; sub-investigators: Thomas Cochrane, Mohammed Salajegheh; primary study coordinator: Kristen Roe; study coordinators (back-up): Katherine Amato, Shirli Toska. University at Buffalo Jacobs School of Medicine and Biomedical Sciences, Buffalo, NY: Principal investigator: Gil Wolfe; sub-investigator: Nicholas Silvestri; study coordinators (back-up): Kara Patrick, Karen Zakalik. California Pacific Medical Center, San Francisco, CA, USA: Principal investigator: Jonathan Katz; sub-investigator: Robert Miller; study coordinators: Marguerite Engel, Dallas Forshew. Carolinas HealthCare System, Charlotte, NC, USA: Principal investigator: Elena Bravver; sub-investigators: Benjamin Brooks, Mohammed Sanjak; study coordinators: Sarah Plevka, Maryanne Burdette; Scott Cunningham, Mohammad Sanjak; study coordinators (back-up): Megan Kramer, Joanne Nemeth, Clara Schommer (regulator coordinator), Scott Tinerney. Duke University Health System, Durham, NC, USA: Principal investigator: Vern Juel; sub-investigators: Jeffrey Guptill, Lisa Hobson-Webb, Janice Massey; primary study coordinators: Kate Beck, Donna Carnes; study coordinator: John Loor; study coordinator (back-up): Amanda Anderson. Indiana University, Indianapolis, IN, USA: Principal investigator: Robert Pascuzzi; Subinvestigators: Cynthia Bodkin, John Kincaid, Riley Snook; primary study coordinator: Sandra Guinrich; study coordinator (back-up): Angela Micheels. Johns Hopkins University School of Medicine, Baltimore, MD, USA: Principal investigator: Vinay Chaudhry; sub-investigator: Andrea Corse; primary study coordinator: Betsy Mosmiller; study coordinator (back-up): Andrea Kelley. Lahey Hospital and Medical Center - Burlington, Burlington, MA, USA: Principal investigator: Doreen Ho; sub-investigators: Jayashri Srinivasan, Michael Vytopil; primary study coordinators: Jordan Jara, Nicholas Ventura; study coordinators: Cynthia Carter, Craig Donahue, Carol Herbert, Stephanie Scala, Elaine Weiner; study coordinator (back-up): Sharmeen Alam. Las Vegas Clinic, Las Vegas, NV, USA: Principal investigator: Jonathan McKinnon; sub-investigators: Laura Haar, Naya McKinnon; study coordinators: Karan Alcon, Kaitlyn McKenna, Nadia Sattar; study coordinators (back-up): Kevin Daniels, Dennis Jeffery. Ohio State University Wexner Medical Center, Columbus, OH, USA: Principal investigator: Miriam Freimer; sub-investigators: Joseph Chad Hoyle, John Kissel; study coordinators: Julie Agriesti, Sharon Chelnick, Louisa Mezache, Colleen Pineda; study coordinator (back-up): Filiz Muharrem. Oregon Health and Science University, Portland, OR, USA: Principal investigators: Chafic Karam, Julie Khoury; previous principal investigator: Tessa Marburger; sub-investigator: Harpreet Kaur; primary study coordinator: Diana Dimitrova. Southern Illinois University School of Medicine, Springfield, IL, USA: Principal investigator: James Gilchrist; sub-investigator: Brajesh Agrawal, Mona Elsayed; primary study coordinator: Stephanie Kohlrus; study coordinators: Angela Andoin, Taylor Darnell; study coordinators (back-up): Laura Golden, Barbara Lokaitis, Jenna Seelbach. Stanford University School of Medicine, Stanford, CA, USA: Principal investigator: Srikanth Muppidi; sub-investigators: Neelam Goyal, Sarada Sakamuri, Yuen T So; study coordinators: Shirley Paulose, Sabrina Pol, Lesly Welsh. The University of Texas Health Science Center at San Antonio, San Antonio, TX, USA: Principal investigator: Ratna Bhavaraju-Sanka; subinvestigator: Alejandro Tobon Gonzalez; study coordinators: Lorraine Dishman, Floyd Jones; study coordinators (back-up): Anna Gonzalez, Patricia Padilla, Amy Saklad, Marcela Silva. The University of Texas Southwestern Medical Center, Dallas, TX, USA: Principal investigator: Sharon Nations; sub-investigator: Jaya Trivedi; study coordinator: Steve Hopkins. University of Alabama at Birmingham Medicine, Birmingham, AL, USA: Principal investigator: Mohamed Kazamel; previous principal investigator: Mohammad Alsharabati; sub-investigator: Liang Lu, Kenkichi Nozaki; study coordinator: Sandi MumfreyThomas; study coordinator (back-up): Amy Woodall. University of California-Irvine, Irvine, CA, USA: Principal investigator: Tahseen Mozaffar; sub-investigators: Tiyonnoh Cash, Namita Goyal, Gulmohor 
Roy; study coordinator: Veena Mathew; study coordinators (back-up): Fatima Maqsood, Brian Minton. University of California-San Francisco-Fresno, CA, USA: Principal investigator: H. James Jones; previous principal investigator: Jeffrey Rosenfeld; study coordinator: Rebekah Garcia; study coordinators (back-up): Laura Echevarria, Sonia Garcia. University of Florida Health Jacksonville, Jacksonville, FL, USA: Principal investigator: Michael Pulley; sub-investigators: Shachie Aranke, Alan Ross Berger, Jaimin Shah; primary study coordinators: Yasmeen Shabbir, Lisa Smith; study coordinator: Mary Varghese; study coordinator (back up): Yasmeen Shabbir. University of Iowa Children's Hospital, Iowa City, IA, USA: Principal investigator: Laurie Gutmann; sub-investigators: Ludwig Gutmann, Nivedita Jerath, Christopher Nance, Andrea Swenson; primary study coordinator: Heena Olalde; study coordinator: Nicole Kressin; study coordinator (back-up): Jeri Sieren. University of Kansas Medical Center, Kansas City, KS, USA: Principal investigator: Richard Barohn; sub-investigators: Mazen Dimachkie, Melanie Glenn, April McVey, Mamatha Pasnoor, Jeffery Statland, Yunxia Wang; primary study coordinator: Tina Liu; study coordinators: Kelley Emmons, Nicole Jenci, Jerry Locheke; study coordinators (back-up): Alex Fondaw, Kathryn Johns, Gabrielle Rico, Maureen Walsh; lead evaluator trainer: Laura Herbelin. University of Maryland Medical Center, Baltimore, MD, USA: Principal investigator: Charlene Hafer-Macko; sub-investigators: Justin Kwan, Lindsay Zilliox; primary study coordinators: Karen Callison, Valerie Young; study coordinator: Beth DiSanzo; study coordinator (back-up): Kerry Naunton. University of Miami Miller School of Medicine, Miami, FL, USA: Principal investigator: Michael Benatar; sub-investigators: Martin Bilsker, Khema Sharma; primary study coordinators: Anne Cooley, Eliana Reyes; study coordinators: Sara-Claude Michon, Danielle Sheldon; study coordinator (back-up): Julie Steele. University of North Carolina Medical Center, Chapel Hill, NC, USA: Principal investigator: James Howard, Jr; sub-investigator: Chafic Karam, Rebecca Traub; study coordinator: Manisha Chopra. University of South Florida, Tampa, FL, USA: Principal investigator: Tuan Vu; sub-investigators: Lara Katzin, Terry McClain; study coordinator: Brittany Harvey; study coordinators (back-up): Adam Hart, Kristin Huynh. University of Southern California, Los Angeles, CA, USA: Principal investigator: Said Beydoun; sub-investigators:Amaiak Chilingaryan, Victor Doan, Brian Droker, Hui Gong, Sanaz Karimi, Frank Lin, Terry McClain, Krishna Polaka, Akshay Shah, Anh Tran; study coordinator: Salma Akhter; study coordinator (back-up): Ali Malekniazi. University of Vermont Medical Center, Burlington, VT, USA: Principal investigator: Rup Tandan; sub-investigator: Michael Hehir, Waqar Waheed; primary study coordinator: Shannon Lucy. University of Washington, Seattle, WA, USA: Principal investigator: Michael Weiss; sub-investigator: Jane Distad; primary study coordinator: Susan Strom; study coordinators: Sharon Downing, Bryan Kim. Wesley Neurology Clinic, PC, Cordova, TN, USA: Principal investigator: Tulio Bertorini; sub-investigators: Thomas Arnold, Kendrick Henderson, Rekha Pillai; primary study coordinator: Ye Liu; study coordinator: Lauren Wheeler; study coordinators (back-up): Jasmine Hewlett, Mollie Vanderhook. Yale University, New Haven, CT, USA: Principal investigator: Richard Nowak; sub-investigators: Daniel Dicapua, Benison Keung, Aditya Kumar, Huned Patwa, Kimberly Robeson, Irene Yang; study coordinator: Joan Nye; study coordinator (back-up): Hong Vu.

Author contributions Study design and conceptualization: James F. Howard, Jr, Kenji P. Fujita, Fanny O'Brien; major role in data acquisition, data analysis and interpretation, drafting and revising the manuscript for intellectual content, final approval of the manuscript: all authors.

Data availability Qualified academic investigators may request participant-level, de-identified clinical data and supporting documents (statistical analysis plan and protocol) pertaining to this study. Further details regarding data availability, instructions for requesting information and our data disclosure policy are available on the Alexion website (https ://alexion.com/research-development).

\section{Compliance with ethical standards}

Conflicts of interest This work was funded by Alexion Pharmaceuticals. J.V. has received research and travel support, and/or speaker honoraria from Alexion Pharmaceuticals and Sanofi/Genzyme, and has served on advisory boards or as a consultant for Asklepios Biopharmaceuticals, Audentes Therapeutics, Novartis Pharma AG, PTC Therapeutics, Roche, Sanofi/Genzyme, Santhera Pharmaceuticals, Sarepta Therapeutics, and Stealth Biotherapeutics within the past 3 years. S.J. is a member of an international advisory board for Alexion Pharmaceuticals, has been an advisory board member for Alnylam Pharmaceuticals and Argenx BVBA, has received speaker fees from Terumo BCT, and has received research support from the Wellcome Trust Clinical Research Facility and Centre for Rare Diseases at the University Hospitals Birmingham, UK. K.P.F. was employed by and owns stock in Alexion Pharmaceuticals and is employed by Alnylam Pharmaceuticals. F.O'B. is employed by, and owns stock in, Alexion Pharmaceuticals. J.F.H. has received research support from Alexion Pharmaceuticals, argenx BVBA, the Centers for Disease Control and Prevention (Atlanta, GA, USA), the Muscular Dystrophy Association, the National Institutes of Health (including the National Institute of Neurological Disorders and Stroke and the National Institute of Arthritis and Musculoskeletal and Skin Diseases) and Ra Pharmaceuticals; has received honoraria from Alexion Pharmaceuticals; and has received non-financial support from Alexion Pharmaceuticals, argenx BVBA, Ra Pharmaceuticals and Toleranzia.

Ethical approval This study was approved by the appropriate ethics committees and has been performed in accordance with the ethical standards laid down in the 1964 Declaration of Helsinki and its later amendments. All participants gave their informed consent prior to inclusion in the study.

Open Access This article is licensed under a Creative Commons Attribution 4.0 International License, which permits use, sharing, adaptation, distribution and reproduction in any medium or format, as long as you give appropriate credit to the original author(s) and the source, provide a link to the Creative Commons licence, and indicate if changes were made. The images or other third party material in this article are included in the article's Creative Commons licence, unless indicated otherwise in a credit line to the material. If material is not included in the article's Creative Commons licence and your intended use is not permitted by statutory regulation or exceeds the permitted use, you will need to obtain permission directly from the copyright holder. To view a copy of this licence, visit http://creativecommons.org/licenses/by/4.0/.

\section{References}

1. Suh J, Goldstein JM, Nowak RJ (2013) Clinical characteristics of refractory myasthenia gravis patients. Yale J Biol Med $86: 255-260$

2. Buzzard KA, Meyer NJ, Hardy TA, Riminton DS, Reddel SW (2015) Induction intravenous cyclophosphamide followed by maintenance oral immunosuppression in refractory myasthenia gravis. Muscle Nerve 52:204-210. https://doi.org/10.1002/ mus. 24536 
3. Grob D, Brunner N, Namba T, Pagala M (2008) Lifetime course of myasthenia gravis. Muscle Nerve 37:141-149. https://doi. org/10.1002/mus.20950

4. Engel-Nitz NM, Boscoe A, Wolbeck R, Johnson J, Silvestri NJ (2018) Burden of illness in patients with treatment refractory myasthenia gravis. Muscle Nerve 58:99-105. https://doi. org/10.1002/mus.26114

5. Drachman DB, Adams RN, Hu R, Jones RJ, Brodsky RA (2008) Rebooting the immune system with high-dose cyclophosphamide for treatment of refractory myasthenia gravis. Ann N Y Acad Sci 1132:305-314. https://doi.org/10.1196/annals.1405.033

6. Silvestri NJ, Wolfe GI (2014) Treatment-refractory myasthenia gravis. J Clin Neuromuscul Dis 15:167-178. https://doi. org/10.1097/CND.0000000000000034

7. Nowak RJ, Dicapua DB, Zebardast N, Goldstein JM (2011) Response of patients with refractory myasthenia gravis to rituximab: a retrospective study. Ther Adv Neurol Disord 4:259-266. https://doi.org/10.1177/1756285611411503

8. Boscoe AN, Xin H, L'Italien GJ, Harris LA, Cutter GR (2019) Impact of refractory myasthenia gravis on health-related quality of life. J Clin Neuromuscul Dis 20:173-181. https://doi.org/10.1097/ CND.0000000000000257

9. Conti-Fine BM, Milani M, Kaminski HJ (2006) Myasthenia gravis: past, present, and future. J Clin Invest 116:2843-2854. https://doi.org/10.1172/JCI29894

10. Lindstrom JM, Seybold ME, Lennon VA, Whittingham S, Duane DD (1976) Antibody to acetylcholine receptor in myasthenia gravis. Prevalence, clinical correlates, and diagnostic value. Neurology 26:1054-1059

11. Mantegazza R, Pareyson D, Baggi F, Romagnoli P, Peluchetti D, Sghirlanzoni A et al (1988) Anti AChR antibody: relevance to diagnosis and clinical aspects of myasthenia gravis. Ital J Neurol Sci 9:141-145

12. Rother RP, Rollins SA, Mojcik CF, Brodsky RA, Bell L (2007) Discovery and development of the complement inhibitor eculizumab for the treatment of paroxysmal nocturnal hemoglobinuria. Nat Biotechnol 25:1256-1264. https://doi.org/10.1038/nbt1344

13. Howard JF Jr, Utsugisawa K, Benatar M, Murai H, Barohn RJ, Illa I et al (2017) Safety and efficacy of eculizumab in anti-acetylcholine receptor antibody-positive refractory generalised myasthenia gravis (REGAIN): a phase 3, randomised, double-blind, placebocontrolled, multicentre study. Lancet Neurol 16:976-986. https:// doi.org/10.1016/S1474-4422(17)30369-1

14. Muppidi S, Utsugisawa K, Benatar M, Murai H, Barohn RJ, Illa I et al (2019) Long-term safety and efficacy of eculizumab in generalized myasthenia gravis. Muscle Nerve 60:14-24. https:// doi.org/10.1002/mus.26447

15. Wolfe GI, Herbelin L, Nations SP, Foster B, Bryan WW, Barohn RJ (1999) Myasthenia gravis activities of daily living profile. Neurology 52:1487-1489
16. Burns TM, Conaway MR, Cutter GR, Sanders DB (2008) Less is more, or almost as much: a 15-item quality-of-life instrument for myasthenia gravis. Muscle Nerve 38:957-963. https://doi. org/10.1002/mus.21053

17. Burns TM, Grouse CK, Conaway MR, Sanders DB (2010) Construct and concurrent validation of the MG-QOL15 in the practice setting. Muscle Nerve 41:219-226. https://doi.org/10.1002/ mus.21609

18. Muppidi S, Wolfe GI, Conaway M, Burns TM, MG Composite and MG-QOL15 Study Group (2011) MG-ADL: still a relevant outcome measure. Muscle Nerve 44:727-731. https://doi. org/10.1002/mus. 22140

19. Howard JF Jr, Freimer M, O’Brien F, Wang JJ, Collins SR, Kissel JT et al (2017) QMG and MG-ADL correlations: study of eculizumab treatment of myasthenia gravis. Muscle Nerve 56:328-330. https://doi.org/10.1002/mus.25529

20. Vissing J, O’Brien F, Wang JJ, Howard JF Jr (2018) Correlation between myasthenia gravis-activities of daily living (MG-ADL) and quantitative myasthenia gravis (QMG) assessments of antiacetylcholine receptor antibody-positive refractory generalized myasthenia gravis in the phase 3 regain study. Muscle Nerve 58:E21-E22. https://doi.org/10.1002/mus.26152

21. Hillmen P, Young NS, Schubert J, Brodsky RA, Socie G, Muus P et al (2006) The complement inhibitor eculizumab in paroxysmal nocturnal hemoglobinuria. N Engl J Med 355:1233-1243. https ://doi.org/10.1056/NEJMoa061648

22. Legendre CM, Licht C, Muus P, Greenbaum LA, Babu S, Bedrosian C et al (2013) Terminal complement inhibitor eculizumab in atypical hemolytic-uremic syndrome. N Engl J Med 368:2169_ 2181. https://doi.org/10.1056/NEJMoa1208981

23. Licht C, Greenbaum LA, Muus P, Babu S, Bedrosian CL, Cohen DJ et al (2015) Efficacy and safety of eculizumab in atypical hemolytic uremic syndrome from 2-year extensions of phase 2 studies. Kidney Int 87:1061-1073. https://doi.org/10.1038/ ki.2014.423

24. Socie G, Caby-Tosi MP, Marantz JL, Cole A, Bedrosian CL, Gasteyger $C$ et al (2019) Eculizumab in paroxysmal nocturnal haemoglobinuria and atypical haemolytic uraemic syndrome: 10-year pharmacovigilance analysis. Br J Haematol 185:297-310. https:// doi.org/10.1111/bjh. 15790

25. Zuber J, Fakhouri F, Roumenina LT, Loirat C, Fremeaux-Bacchi V, French Study Group for a HCG (2012) Use of eculizumab for atypical haemolytic uraemic syndrome and C3 glomerulopathies. Nat Rev Nephrol 8:643-657. https://doi.org/10.1038/nrnep h.2012.214 